\section{Glycine transporter inhibitors}

Current treatment of schizophrenia offers a wide variety of psychopharmaceuticals that are licensed as first-line treatments for this disorder. However, if we consider their dominant mechanism of action, choice eventually narrows, in some instances considerably. In light of the facts that one third of patients do not respond to standard treatment and that current treatment options do not affect all schizophrenia symptom dimensions, it is not hard to conclude that there is an unsatisfied need which should drive the development of new treatment modalities focusing on other neurotransmitter systems beside dopamine and serotonin [1].

There have been recent discoveries in terms of convergent and convincing evidence from pharmacological, autoimmune, and genetic studies which have confirmed that impaired glutamatergic N-methyl-D-aspartate (NMDA) receptor function can produce psychotic symptoms and that it is involved in the pathogenesis of schizophrenia. Glycine transporters 1 (GlyT1) play a key role in terminating the activity of glycine, which is released into synapses from glial cells to bind and act at a binding site of the NMDA receptor. Glycine, with glutamate, is indispensable for activation of NMDA receptors. Consequently, blocking the glycine transporter increases the synaptic availability of glycine and thus improves NMDA neurotransmission. Furthermore, this enhances GABA neurotransmission in the cortex, which in turn leads to a reduction in glutamatergic neurotransmission. Reduced glutamate release in the ventral tegmental area does not lead to excessive stimulation of the mesolimbic dopamine pathway. The terminal goal of GlyT1 inhibition is the abrogation of impaired NMDA receptor functionality. Exactly in this way GlyT1 inhibition could potentially contribute to the reduction of cognitive and negative symptoms of schizophrenia with the reduction of positive symptoms. GlyT1 inhibitors are also called selective glycine reuptake inhibitors or SGRI, in analogy to drugs that inhibit the reuptake of other neurotransmitters, such as serotonin reuptake inhibitors which acts upon the serotonin transporter (SSRI) [1,2].

Several GlyT1 inhibitors are currently in the clinical testing phase, including bitopertin, whose chemical structure is shown in Figure 1. We would highlight a clinical trial program 
called "SearchLyte," as one of the significant studies related to bitopertin, which consisted of six Phase III studies. Three studies targeted the ability to treat patients with persistent negative symptoms. The other three studies were designed to determine the ability of the drug to reduce the positive symptoms of patients who did not respond to the currently available antipsychotic. Two trials were discontinued after analysis and the remaining trials did not meet their primary end goals with the exception of the NightLyte study. The aforementioned study included patients with a suboptimal response to treatment with prior antipsychotics and found that the addition of bitopertin at a dose of $10 \mathrm{mg}$ significantly improved the PANSS scores. While phase III trials focused on the additional use of bitopertin, a phase II / III monotherapy trial was also conducted. It included patients with acute exacerbation and compared patients treated with bitopertin to placebo and the active control group using olanzapine. A trend of improvement in overall PANSS score was detected for both bitopertin (30 $\mathrm{mg}$ ) and olanzapine, which was the main endpoint of the study. However, this was not significantly different from placebo for either drug. Such a result was considered a failed study, but bitopertin showed a significant improvement in PANSS assessment and an increased willingness of treated patients for demission from hospital treatment compared to placebo. Such, in some aspects, somewhat disappointing results of the bitopertin trial could be explained by a number of reasons, for example, the first being the possibility of a particularly pronounced placebo response effect. Namely, placebo reactions are of particular concern in studies of negative symptoms that may be primary or secondary. Primary negative symptoms are inherent to the disease and therefore potentially respond to interventions targeting underlying biological factors, while secondary negative symptoms arise from factors caused by social isolation and stigma. The latter are likely to respond to psychosocial intervention and may thus be enhanced by regular social interactions, including regular assessments and medical examinations as part of a clinical trial, thus contributing to a markedly more prominent placebo effect. Phase III studies were conducted in chronic patients who likely developed secondary negative symptoms. Another problem with inclusion of chronic patients in phase III studies is the fact that there is evidence of glutamate abnormalities which are more pronounced in the early stages of the disease and that drugs targeting this system can only have an effect on patients in the early stages of the disease. These considerations suggest that future studies should focus on patients in the early stages of the disease $[3,4]$.

Another prominent Gly'T1 inhibitor is Nmethylglycine, which is also known as sarcosine. Sarcosine has been the subject of numerous, although predominantly small, schizophrenia studies that have shown its superiority over placebo, either alone or as augmentative therapy. According to some reports, sarcosine improved negative, cognitive and depressive symptoms of schizophrenia, including symptoms such as alogia and blunt affect $[5,6]$. However, further evidence is needed in order to confirm these facts. The standard dose of sarcosine used in clinical studies was usually 2 grams per day, although there are recommendations that titration should be performed on a gradual basis $[5,6]$. The chemical structure of sarcosine is shown in Figure 2. Sarcosine otherwise naturally occurs in various foods and is sold without re- 
<smiles>C[C@@H](Oc1ccc(S(C)(=O)=O)cc1C(=O)N1CCN(c2ncc(C(F)(F)F)cc2F)CC1)C(F)(F)F</smiles>

Figure 1. Chemical structure of bitopertin

striction. It is extensively promoted on the internet as a supplement for "brain health" and for various mental health problems. Even in the formulation of dietary supplements there is reasonable evidence that it has a true pharmacological effect [7].

Several other GlyT1 inhibitors are also in the preclinical study phase, such as SSR 504734, SSR 241586, JNJ17305600, and PF03463275 (2).

It is important to note that several independent lines of research have demonstrated that pharmacological inhibition of glycine reuptake may also be relevant for the treatment of various other clinical conditions, including depression, anxiety disorders, obsessive compulsive disorder, alcohol dependence, epilepsy and pain. This could be explained by the physiological impact of glycine reuptake inhibition on either inhibitory

\section{References}

1. Stahl SM. Stahlovi temelji psihofarmakologije. Jastrebarsko: Naklada Slap; 2017.

2. Karlović D, Peitl V, Silić A. Shizofrenije. Jastrebarsko: Naklada Slap; 2019.

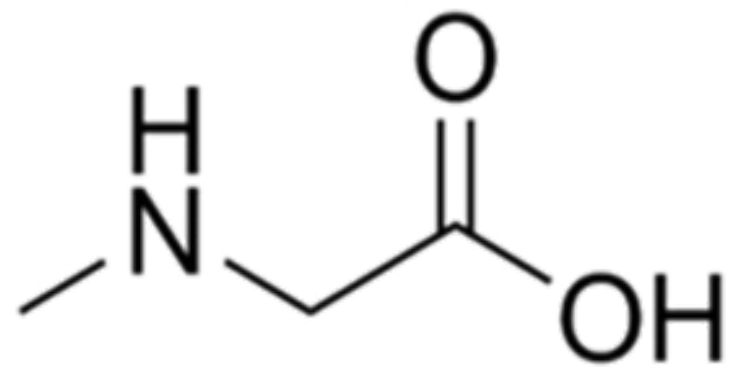

Figure 2. Chemical structure of sarcosine

glycinergic neurotransmission or NMDA receptor-dependent excitatory glutamatergic neurotransmission. However, none of the proposed clinical applications can integrate the expected neuropharmacological effects of selective inhibitors of glycine reuptake, leading to the conclusion that the dual action of glycine in the nervous system may explain why none of these approaches have yielded clinically ready drugs [2, 8-12].

In conclusion, we cheer on and hope for further investigations of GlyT1 inhibitors, focusing on their potential to improve NMDA receptor functionality, as well as clinically beneficial changes in symptom expression. Especially in terms of negative and cognitive symptomatology of schizophrenia, but also in other potential clinical applications.

Assistant Professor Vjekoslav Peitl, MD, PhD

Darko Vlahović, MD

3. Singer P, Yee BK. Pharmacotherapy Through the Inhibition of Glycine Transporters: An Update on and Beyond Schizophrenia. In: Gargiulo P, Mesones-Arroyo H, (eds). Psychiatry and Neuroscience Update - Vol. II. Cham, Switzerland: Springer; 2017. 
4. Beck K, Javitt DC, Howes OD. Targeting glutamate to treat schizophrenia: lessons from recent clinical studies. Psychopharmacology. 2016;233:2425-8.

5. Strzelecki D, Urban-Kowalczyk M, Wysokinski A. Serum levels of TNF-alpha in patients with chronic schizophrenia during treatment augmentation with sarcosine (results of the PULSAR study). Psychiatry Res. 2018;268:447-53.

6. Amiaz R, Kent I, Rubinstein K, Sela B, Javitt D, Weiser M. Safety, tolerability and pharmacokinetics of open label sarcosine added on to anti-psychotic treatment in schizophrenia - preliminary study. Isr J Psychiatry Relat Sci. 2015;52:12-16.

7. Curtis D. A possible role for sarcosine in the management of schizophrenia. Br J Psychatry. 2019;215:697-698.

8. Huang CC, Wei IH, Huang CL, Chen KT, Tsai $\mathrm{MH}$, Tsai $\mathrm{P}$, et al. Inhibition of glycine transport-
er-I as a novel mechanism for the treatment of depression. Biol Psychiatry. 2013;74:734-41.

9. Wu PL, Tang HS, Lane HY, Tsai CA, Tsai GE. Sarcosine therapy for obsessive compulsive disorder: a prospective, open-label study. J Clin Psychopharmacol. 2011;31:369-74.

10. Socała K, Nieoczym D, Rundfeldt C, Wlaź P. Effects of sarcosine, a glycine transporter type 1 inhibitor, in two mouse seizure models. Pharmacol Rep. 2010;62:392-7.

11. Centeno MV, Mutso A, Millecamps M, Apkarian AV. Prefrontal cortex and spinal cord mediated anti-neuropathy and analgesia induced by sarcosine, a glycine-T1 transporter inhibitor. Pain. 2009;145:176-83.

12. Molander A, Lidö HH, Löf E, Ericson M, Söderpalm B. The glycine reuptake inhibitor ORG25935 decreases ethanol intake and preference in male wistar rats. Alcohol Alcohol. 2007;42:11-8. 